\title{
Interactive comment on "Combining satellite data and appropriate objective functions for improved spatial pattern performance of a distributed hydrologic model” by Mehmet C. Demirel et al.
}

\author{
H. Bogena (Referee) \\ h.bogena@fz-juelich.de
}

Received and published: 20 October 2017

This paper deals with an innovative calibration framework that combines temporally aggregated observed spatial patterns with a new spatial performance metric and a flexible spatial parameterisation scheme. An application of the mesoscale Hydrologic Model ( $\mathrm{mHM}$ ) to the Skjern River Basin is used as an example show the effectiveness of the presented calibration framework. This a very timely topic that fits very well to the scope of HESS.

However, the spatial model parameterizing methodology is not well described. Both the root fraction coefficient and the PET correction factor parameterizations should be

Printer-friendly version

Discussion paper 
presented in more detail including graphical presentations of the underlying relationships.

For these reasons, my recommendation is to accept this manuscript with minor revisions. I have provided specific comments and suggestions for improvement below.

Specific comments:

P3L7: Which models?

P3L16-32: This section is redundant with the method section and should be shortened.

\section{P5L6 Delete "s"}

P5L9-11: How accurate are the AET maps, e.g. in relation to continuous ECmeasurements?

P5L30-31: Why should this procedure accelerate model runs?

P6L8: "stretch the spatial contrast" of what?

P6L8: “. . based on soil and vegetation properties..."

P6L11: Please change "domain-specific" into "site-specific" or "local"

P6L11-12: According Feddes et al. (2001) and others, the root fraction coefficient is a vegetation dependent coefficient. Please add more information on why your assumption that this coefficient can be explained by field capacity is justified.

P6L14-15: At this point it unclear why these parameterizations increase the model freedom. Please reorganise the text in a more comprehensively way.

P6L19-24: It took me some time to understand your method, also because the assumption that the root fraction is linked to $F C$ is counterintuitive. For a better understanding of your method, it would be helpful to graphically show the relationship between FC and rooting depth based on the soil database.

Printer-friendly version

Discussion paper 
P6L22: Why do you restrict your method to pervious non-forested areas?

P6L22: Please change "domain-specific" into "site-specific" or "local"

P7L2: Why should soil properties of sandy soils impede root development?

P7L2-3: Which parameters and why is a fine vertical discretization more effective?

P7L5-7: By changing the root fraction parameters for maximum and minimum FC, the relationship between $\mathrm{FC}$ and rooting depth will be changed. In the extreme case, both parameters have the same value, which means that there is no relationship at all. Did you check whether the optimised model still provides realistic relationships between FC and rooting depth? In addition, it is unclear, how you derived FC values from your digital soil map. Typically, one would sum up the horizontal FC values down to the certain soil depth (e.g. rooting depth). Please provide additional information.

P7L16-17: You should not mention equations that have not been already introduced.

P7L21-25: The section needs to be rewritten in a more comprehensible way.

P7L26: It is how this equation was derived and why it should be "physically meaningful". In addition, it is unclear how the DSF parameter is used to correct ET_ref.

P7L28: The previous sections also belong to "Methods".

P9L1: Only accepted paper should be used a reference.

P9L4-9: This section can be omitted.

P9L24-26: Is this statement relevant for this work or Koch et al., 2017a

P9L34: Why are you using the "same" cloud-free days?

P10L5: Either use the Greek symbol for phi or "phi" (here and elsewhere)

Printer-friendly version

P10L22: Delete "and"

Discussion paper

P10L33-34: I wonder why the parameter "root fraction" for forest area is listed and not 
for the impervious non-forest areas, since the latter was used for the spatial parameterization.

P10L34: "(SPAEF column in Tab. 2)"

P11L9-10: Why are you using a second model warm-up period (2005-2008)? This is also not mentioned in the text.

P12L18-20: However, it should be noted that the uncertainty of the spatial AET information from remote sensing is typically larger than the runoff measurements.

P12L28: Delete "that"

P13L2-3: This indicates that the spatial AET information either has large uncertainties or that it has very imitated information on the subsurface properties due to extensive irrigation in the catchment. Please discuss.

P15L13: Does Figure 4 present a certain year or monthly averages of several years?

P15L14: The differences seem to vary also from month to month. You should quantify the differences in AET patters, e.g. by presenting the variances or variograms.

P17L21: Awkward sentence. Please reformulate.

P17L25: "This was..."

P617L31-32: Unclear why the sandy soil texture should restrict the model parametrisation method.

P617L32: Please change "domain-specific" into "site-specific" or "local"

P18L23: "has proven"

Figures and Tables:

Figure 1: The RS-AET scale should be reversed (red should indicate high AET values)

Table 1: Columns 3, 4 and 5 should be removed as they provide only limited informa- 
tion, which is already presented in the text.

Additional literature

Feddes, R.A., H. Hoff, M. Bruen, T. Dawson, P. de Rosnay, P. Dirmeyer, R.B. Jackson, P. Kabat, A. Kleidon, A. Lilly, and A.J. Pitman, 2001: Modeling Root Water Uptake in Hydrological and Climate Models. Bull. Amer. Meteor. Soc., 82, 2797-2809.

Interactive

comment

Interactive comment on Hydrol. Earth Syst. Sci. Discuss., https://doi.org/10.5194/hess-2017570, 2017. 